corrected intensities can only be regarded as approximate.

The experimental intensities normalized in the above manner are presented in Table I. The errors in the table reflect $90 \%$ confidence limits, assuming no systematic errors are present.

For charge +3 the intensity of $R_{111}$ is just the value one would expect from only $R_{111}{ }^{+3} \gamma$, i.e., essentially all recoils followed by conversion electrons have higher charges. We assumed then that the $0,+1$, and +2 recoils were composed of only $R_{0}$ and $R_{111} \gamma$, which was deduced from the known $111 \mathrm{keV} \gamma$ ray intensity $(4.8 \%)$. The resulting charge distributions of the $R_{0}$ recoils and the $R_{111} e$ recoils are plotted in Fig. 2 .

Szucs and Delfosse $\mathrm{e}^{3}$ studied $\mathrm{Po}^{216}$ recoils from
$\mathrm{Rn}^{220}$ alpha decay and found the maximum in the charge distribution occurring at zero, in contrast to our results with $\operatorname{Th}^{226} R_{0}$ where the maximum intensity occurs at a charge of +1 . The charge distribution of the $\mathrm{Th}^{226} R_{111}$ e recoils is roughly similar to that found by Snell and Pleasonton ${ }^{4}$ for $\mathrm{Xe}^{131}$.

*This work was performed under the auspices of the U. S. Atomic Energy Commission.

${ }^{1}$ Frank Asaro and I. Perlman, Phys. Rev. 104, 91 (1956).

${ }^{2}$ Carl P. Ruiz, University of California Radiation Laboratory Report No. UCRL-9511, 1961 (unpublished).

${ }^{3}$ S. Szucs and J. M. Delfosse, Phys. Rev. Letters $\underline{15}$, 163 (1965).

${ }^{4}$ A. H. Snell and F. Pleasonton, Phys. Rev. $\underline{111}, 1338$ (1958).

\title{
NUCLEAR GIANT QUADRUPOLE RESONANCE
}

\author{
R. Ligensa and W. Greiner \\ Institut für Theoretische Physik, Universität Frankfurt, Frankfurt am Main, Germany
}

and

M. Danos

National Bureau of Standards, Washington, D. C.

(Received 24 January 1966)

We have extended the description of the giant quadrupole resonance of the deformed nuclei ${ }^{1,2}$ to include the coupling between the different collective modes in a quantum mechanical treatment, i.e., we have developed the quantum hydrodynamics of the nucleus including all modes, viz. the rotations, the surface vibrations, and the giant resonance oscillations, up to quadrupole multipolarity. The interactions with the odd particle are also taken into account. The total Hamiltonian can be written

$$
\begin{gathered}
H=E_{R}\left[I^{2}-I_{3}{ }^{2}-j_{3}{ }^{2}-d_{3}{ }^{2}-q_{3}{ }^{2}\right]+\left(\hbar^{2} / 16 B \eta^{2}\right)\left[\left(I_{3}-j_{3}\right)^{2}+q_{3}{ }^{2}+d_{3}{ }^{2}-1\right]+\left(\hbar^{2} / 8 B \eta^{2}\right)\left(I_{3}-j_{3}\right)\left(d_{3}+q_{3}\right) \\
-\left(\hbar^{2} / 2 B\right)\left(\partial^{2} / \partial \xi^{2}+\frac{1}{2} \partial^{2} / \partial \eta^{2}\right)+\frac{1}{2} C_{0} \xi^{2}+C_{2} \eta^{2}+H_{\mathrm{part}}+\sum_{\mu=-1}^{+1} \hbar \omega_{\mu}^{(1)}\left[1+G_{\mu}^{(1)}\left(\xi+6^{1 / 2} \mu \eta\right)\right] b_{\mu}{ }^{(1)+} b_{\mu}{ }^{(1)} \\
+\sum_{\nu=-2}^{+2} \hbar \omega_{\nu}{ }^{(2)}\left\{1+G_{\nu}{ }^{(2)}\left[\xi-\nu\left(4-\nu^{2}\right)\left(\frac{2}{3}\right)^{1 / 2} \eta\right]\right\} b_{\nu}{ }^{(2)+} b_{\nu}{ }^{(2)} .
\end{gathered}
$$

For $H_{\text {part }}$ the Nilsson-Hamiltonian has been used. $I, j, d$, and $q$ are the angular momentum operators of the nucleus, the odd particle, the giant dipole resonance phonons, and the giant quadrupole resonance phonons, respectively. $\xi$ and $\eta$ describe the $\beta$ and $\gamma$ vibrations of the surface. $b^{(1)}$ and $b^{(2)}$ are the annihilation operators for the dipole and quadrupole oscillation phonons, respectively. The diverse energies and the coupling constants $G_{\nu}{ }^{(L)}$ are given by

$$
\hbar \omega_{\mu}^{(1)}=\hbar \omega_{1}\left[1+0.08(-2)^{-|\mu|} \beta\right]\left[1+(-2)^{-|\mu|}\right]^{-1} \text {, }
$$




$$
\begin{gathered}
G_{\mu}^{(1)}=(-2)^{-|\mu|}\left\{-\left[1+(-2)^{-|\mu|} \beta\right]^{-1}+0.08\left[1+0.08(-2)^{-|\mu|} \beta\right]^{-1}\right\}, \\
\hbar \omega_{\nu}^{(2)}=\hbar \omega_{2}\left[1+0.225\left(\nu^{2}-2\right) \beta\right], \quad G_{\nu}^{(2)}=0.225\left(\nu^{2}-2\right) \beta\left[1+0.225\left(\nu^{2}-2\right) \beta\right]^{-1}, \\
\beta=(5 / 4 \pi)^{1 / 2} \beta{ }_{0}, E_{R}=\hbar^{2} / 2 J_{0} ; \quad E_{\beta}=\hbar\left(C_{0} / B\right)^{1 / 2} ; E_{\gamma}=\hbar\left(C_{2} / B\right)^{1 / 2} .
\end{gathered}
$$

The wave functions of (1) are properly symmetrized products of functions describing the rotations, the $\beta$ vibrations, the $\gamma$ vibrations, the odd particle, the giant dipole phonons, and the giant quadrupole phonons. The formulas for the absorption cross section of the dipole resonances are found in Arenhövel, Greiner, and Danos $^{3}$ and Bramblett et al. ${ }^{4}$ The absorption cross section for the giant quadrupole resonances is

$$
\begin{aligned}
\sigma_{E 2}(E)= & \frac{2 \pi}{5} \frac{N Z}{A} \frac{e^{2}}{\hbar c} \frac{R^{2}}{M c^{2}} \\
& \times(1+\alpha) \sum_{n} \frac{E_{n}{ }^{2} f_{n}^{(2)} / \Gamma_{n}}{\left[\left(E^{2}-E_{n}{ }^{2}\right) / E \Gamma_{n}\right]^{2}+1}
\end{aligned}
$$

where

$$
f_{n}^{(2)}=\frac{2 \pi}{3} \frac{A}{N Z} \frac{2 M}{\hbar^{2}} \frac{E_{n}}{5\left(2 I_{i}+1\right)} \frac{|\langle n\|Q\| i\rangle|^{2}}{R^{2}(1+\alpha)}
$$

are the quadrupole strengths and $Q$ is the quad-

\begin{abstract}
rupole operator. The various giant quadrupole resonances $|n\rangle$ are those solutions of (1) where one quadrupole phonon is excited. There exist five main $E 2$ giant resonances containing most of the quadrupole strengths. They correspond to the excitation of the proton-neutron fluids along the long nuclear axis (one mode), along the short nuclear axes (two modes), and along the directions $\theta=\pi / 4, \varphi$, or $\pi / 2$ (two modes). Because of the interaction with the surface vibrations the various modes split. Some satellites (one surface phonon excited on top of the giant resonances) also contain appreciable quadrupole strengths. The parameters entering the problem are all fixed from the first giant dipole resonance peak and the low-energy spectrum of the nucleus. The positions and the strengths of the various giant quadrupole levels are then completely determined and no free parameters remain in the theory which could influence these quantities.
\end{abstract}

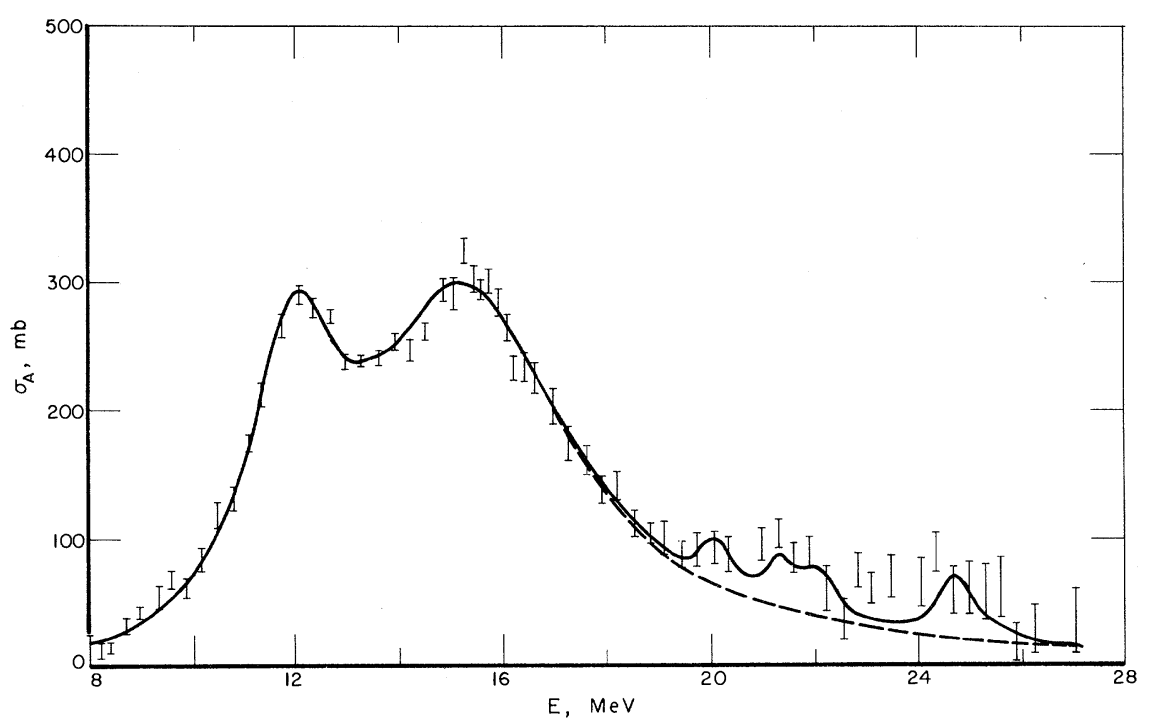

FIG. 1. The total absorption cross section of ${ }^{165} \mathrm{Ho}$. The lines show the theoretical cross sections. The full line is the sum of the dipole and the quadrupole cross sections. The dipole cross section is shown separately by the dotted line. The following parameters were used for the theoretical curve: $\beta_{0}=0.24, E_{\beta}=1.46 \mathrm{MeV}, E_{\gamma}=1.00$ $\mathrm{MeV}, E_{R}=10.52 \mathrm{keV}, E_{0}=12.0 \mathrm{MeV}, \Gamma_{0}=2.3 \mathrm{MeV}, \delta=1.6, \alpha=0.25 ; \Gamma_{Q}=0.6 \mathrm{MeV}$. The experimental points are from Ref. 5 . 


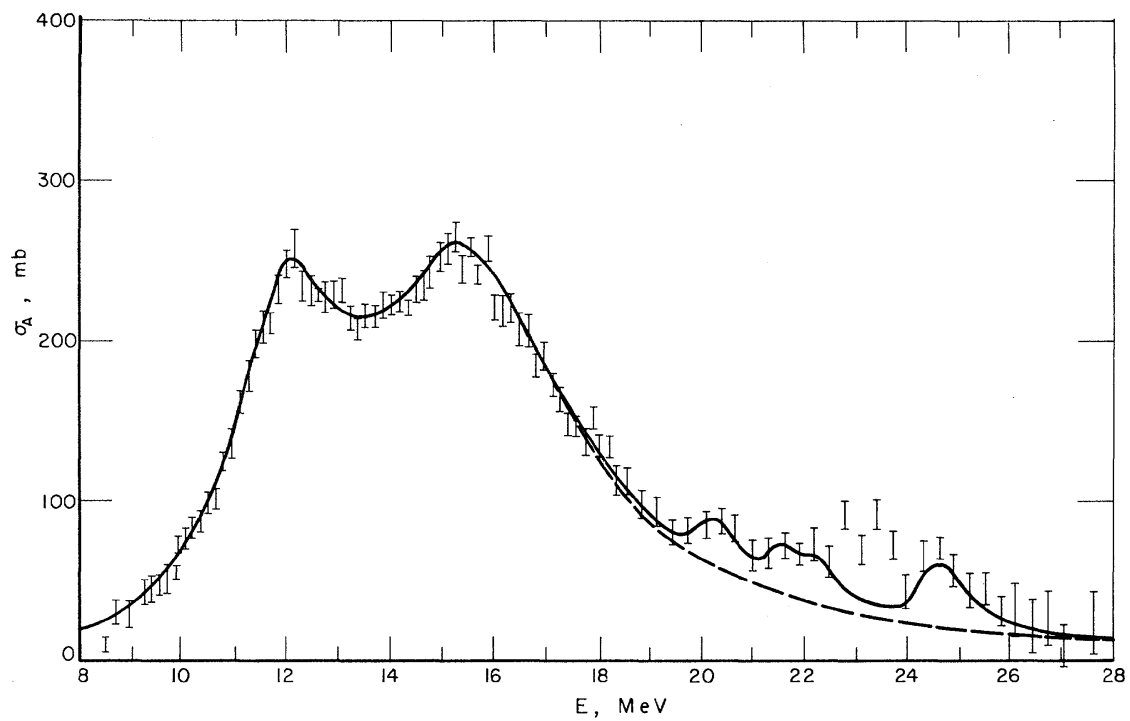

FIG. 2. The same as Fig. 1 for ${ }^{159} \mathrm{~Tb}$. The parameters are $\beta_{0}=0.24, E_{\beta}=1.50 \mathrm{MeV}, E_{\gamma}=1.00 \mathrm{MeV}, E_{R}=11.60$ $\mathrm{keV}, E_{0}=12.0 \mathrm{MeV}, \Gamma_{0}=2.5 \mathrm{MeV}, \delta=1.6, \alpha=0.17, \Gamma_{Q}=0.7 \mathrm{MeV}$. Experiment, Ref. 6 .

We shall compare the theory with the recent Livermore experiments, ${ }^{\mathbf{5}, \mathbf{6}}$ which constitute a large fraction of the available data. The experimental data for ${ }^{165} \mathrm{Ho}$ and ${ }^{159} \mathrm{~Tb}$ are given in Figs. 1 and 2. The dashed line represents the tail of the giant dipole resonance. The absorption cross section of the giant quadrupole resonance, i.e., the cross section which lies above the dashed curve, is shown separately in a larger scale in Figs. 3 and 4 . The theoretical curves were computed assuming a constant width, $\Gamma_{Q}$, for all giant quadrupole states of the nucleus. The widths of the different giant dipole states were taken as $\Gamma_{n}=\Gamma_{0}\left(E_{n} / E_{0}\right)^{\delta}$, i.e., they were taken to vary with a power of

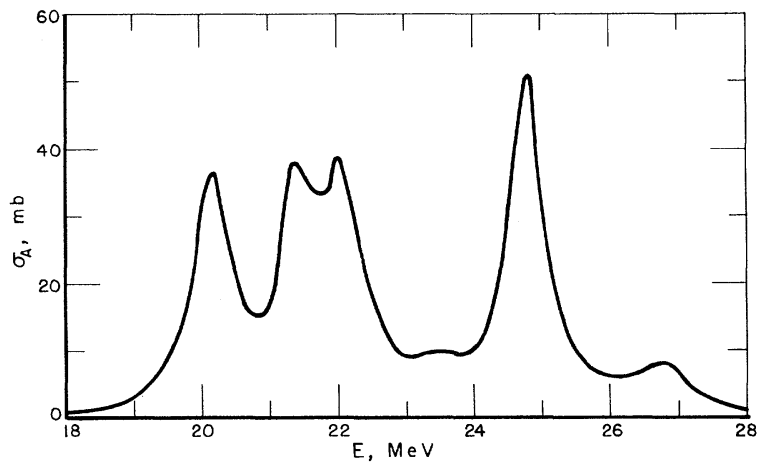

FIG. 3. The theoretical quadrupole absorption cross section for ${ }^{165} \mathrm{Ho}$. the energy of the states. ${ }^{7}$ The full lines in these figures represent the sum of the calculated $E 1$ and the $E 2$ photon absorption cross sections.

The actual shape of the absorption cross section above $17 \mathrm{MeV}$ is subject to some question as a result of the uncertainties in the $(\gamma, 2 n)$ and $(\gamma, 3 n)$ cross sections ${ }^{5,8}$ and the magnitude of a nonresonating component in the total cross section as indicated by the experiments of Ambler, Fuller, and Marshak ${ }^{9}$ as well as those of Mutchler et al. ${ }^{10}$ All of the data are, however, consistent with the absorption cross section at energies above $18 \mathrm{MeV}$ being somewhat above that obtained by extrapolating the electric dipole giant resonance into this energy

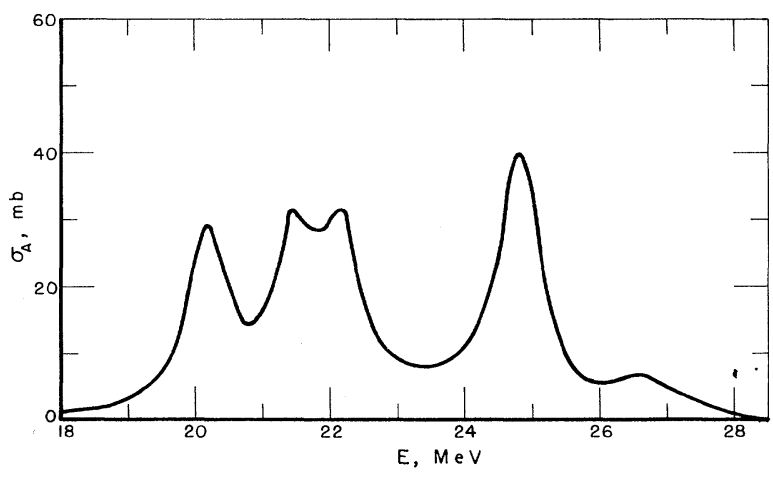

FIG. 4. The theoretical quadrupole absorption cross section for ${ }^{159} \mathrm{~Tb}$. 
region. The existence of $E 2$ absorption in the region of 20-30 MeV has been shown by the earlier experiments of Sorokin et al. ${ }^{11}$ We therefore conclude that the theory is fully compatible with the available experimental data, and we also believe that the observed cross section exceeding the value ascribable to the highenergy tail of the giant dipole resonance is indeed the giant quadrupole resonance.

We acknowledge fruitful discussions with H. Arenhövel and stimulating criticism from E. G. Fuller and E. Hayward.

\footnotetext{
${ }^{1}$ M. Danos, W. Greiner, and B. C. Kohr, Report, University of Freiburg in Braunschweig, 1964 (to be published).

${ }^{2}$ M. Danos, W. Greiner, and B. C. Kohr, Phys. Letters 12, 344 (1964).

${ }^{3}$ M. Danos and W. Greiner, Phys. Rev. 134, B287
}

(1964); Phys. Letters $\underline{8}, 113$ (1964); Phys. Rev. $\underline{138}$, B876 (1965).

${ }^{4}$ H. Arenhövel, W. Greiner, and M. Danos, to be published; see also H. Arenhövel, Dissertation, Universität Frankfurt am Main, 1965, (unpublished).

${ }^{5}$ R. L. Bramblett, J. T. Caldwell, G. F. Auchanpaugh, and S. C. Fultz, Phys. Rev. 129, 2723 (1963).

${ }^{6}$ R. L. Bramblett, J. T. Caldwell, R. R. Harvey, and S. C. Fultz, Phys. Rev. 133, B868 (1964).

${ }^{7}$ M. Danos and W. Greiner, Phys. Rev. 138, B876 (1965).

${ }^{8}$ P. Axel, J. Miller, C. Schuhl, G. Tanan, and C. Tza$\mathrm{ra}$, to be published.

${ }^{9}$ E. Ambler, E. G. Fuller, and H. Marshak, Phys. Rev. 138, B117 (1965).

${ }^{10}$ G. S. Mutchler, W. Bertozzi, S. Kowalski, L. P. Sargent, and W. Turchinetz, Bull. Am. Phys. Soc. 10, 541 (1965), and private communication.

${ }^{11}$ Yu. I Sorokin, V. G. Shevchenko, and B. A. Yur'ev, Zh. Eksperim. i Teor. Fiz. 43, 1600 (1962) [translation: Soviet Phys.-JETP 16, 1127 (1963)].

\section{METHOD TO TEST FOR TIME-REVERSAL INVARIANCE VIOLATION IN $K$ DECAYS* \\ David Cline}

Department of Physics, University of Wisconsin, Madison, Wisconsin (Received 17 January 1966)

Recent evidence for $C P$-invariance violation in $K_{2}{ }^{0}$ decay ${ }^{1}$ has spurred experimental tests of $C P$ and time-reversal invariance in several other physical systems. ${ }^{2}$ In this note we suggest another physical system for which it may be fruitful to test for time-reversal invariance and we report a crude experimental limit on time-reversal invariance violation in this system.

The most general matrix element for $\pi^{+} \pi^{0} \gamma$ decay of the $K^{+}$meson can be written as a sum of three terms ${ }^{3}$ :

$$
M\left(\pi^{+} \pi^{0} \gamma\right)=\alpha f_{1}+\epsilon f_{2}+m f_{3},
$$

where the terms $f_{i}(i=1,2,3)$ represent Lorentzinvariant factors consisting of products of the four-vectors for the system and the terms $\alpha$, $\epsilon$, and $m$ represent form factors corresponding to inner bremsstrahlung, direct electricdipole transitions, and direct magnetic-dipole transitions, respectively. $\alpha$ is also the offmass-shell amplitude for $K^{+} \rightarrow \pi^{+}+\pi^{0}$ decay.

Time-reversal invariance requires that the form factors be relatively real except for finalstate interactions. ${ }^{4}$ Relaxing the requirement of time-reversal invariance and including final- state interactions leads to the substitutions ${ }^{4}$

$$
\begin{gathered}
\alpha \rightarrow \tilde{\alpha} \exp \left[i\left(\delta_{2}+\varphi_{2}\right)\right], \\
\epsilon \rightarrow \tilde{\epsilon} \exp \left[i\left(\delta_{1}+\varphi_{1}\right)\right], \\
m-\tilde{m} \exp \left[i\left(\delta_{1}+\varphi_{1}{ }^{\prime}\right)\right],
\end{gathered}
$$

where $\delta_{2}$ and $\delta_{1}$ are the $T=2$ and $T=1 \pi \pi$ scattering phase shifts, respectively, and $\left(\varphi_{2}, \varphi_{1}, \varphi_{1}{ }^{\prime}\right)$ are time-reversal noninvariance phases.

The positive-pion spectrum for $\pi^{+} \pi^{0} \gamma$ decay is

$$
d B\left(\pi^{+}\right) / d T\left(\pi^{+}\right)=\Gamma_{\pi 2}\left[B+\gamma \cos \Delta I+\left(\gamma^{2}+\beta^{2}\right) E\right],
$$

where $\gamma=\tilde{\epsilon} / \tilde{\alpha}, \beta=\tilde{m} / \tilde{\alpha}$, and $\Delta=\left(\delta_{1}-\delta_{2}\right)+\left(\varphi_{1}-\varphi_{2}\right)$, and where $B, I$, and $E$ are known functions of the pion kinetic energy $T\left(\pi^{+}\right)$. The functions $B, I$, and $E$ are tabulated in Ref. 3. $\Gamma_{\pi 2}$ is the $K_{\pi 2}$ decay rate.

In deriving Eq. (2) a summation over photon polarization removes all cross terms involving the magnetic-dipole transition $(\tilde{m})$. In Eq. (2) the rescattering of pions from inner bremsstrahlung decay has been neglected since the effects are expected to be quite small..$^{5}$ To test $\pi^{+} \pi^{0} \gamma$ decay for time-reversal invariance the phase 